\title{
MODELLING DYNAMIC OPACITY USING PETRI NETS WITH SILENT ACTIONS
}

\author{
Jeremy W. Bryans, Maciej Koutny and Peter Y.A. Ryan \\ School of Computing Science, University of Newcastle, \\ Newcastle upon Tyne, NE1 TRU, U.K.
}

\begin{abstract}
In a previous work, [1], we presented a Petri Net based framework in which various confidentiality properties may be expressed in terms of predicates over system state and abstraction mappings from the reachable states and transitions of the underlying Petri Net. Here we extend that work by generalising these mappings by allowing them to be state dependent. This provides a natural framework in which to model various situations of importance in security, for example key compromise and refresh, downgrading of secrecy labels and conditional anonymity. We also show how global changes in the abstraction mappings can be used to model how some secrecy requirements depend on the status of the observer. We illustrate this by modelling the various flavours of anonymity that arise in the dining cryptographers example.

A further development on the earlier work is to provide a more complete treatment of silent actions. We also discuss the expressiveness of the resulting framework and the decidability of the associated verification problems. ${ }^{1}$
\end{abstract}

Keywords: opacity, non-deducibility, anonymity, Petri nets, observable behaviour, silent actions.

\section{Introduction}

The notion of opacity with respect to a given system predicate, see for example [10], formalises the idea that an observer of a system may never be able to establish the truth of that predicate. As such it appears to be very general and flexible and to allow a wide class of well-established notions of secrecy to be captured.

In a previous paper, [1], we presented a Petri Net based framework in which various opacity properties may be expressed in terms of predicates over system state and abstraction mappings from the reachable states 
and transitions of an underlying Petri Net. in this paper we extend this earlier work in a number of respects:

- Rather than just considering a static abstraction mapping we allow the transition labels to depend on the markings, i.e., the system state.

- We give a more satisfactory treatment of silent actions.

- We introduce a further Petri Net based notion of opacity, namely total opacity.

- We give a more extensive discussion of the notion of anonymity in this framework. In particular we illustrate, using the full dining cryptographers example, how flavours of anonymity may change with the observer viewpoint, i.e., with the abstraction mapping.

- The decidability results of [1] are extended to this richer model.

Our earlier framework allowed us, via the notion of opacity, to capture a number of situations of importance in security but that sit awkwardly with the more familiar information flow concepts such as noninterference [3]. These include, for example, anonymity and encrypted channels in which there is inevitably some partial information flow. The extended framework presented here allows us to go further and capture situations in which the information flow may vary with the state of the system. Note that we can include the state of an adversary in our system model. Thus we can now model key compromise or refresh as well as classification downgrades.

The new form of opacity, total opacity, that we introduce here further allows us to capture the notion of non-inference.

Using the framework of Petri nets gives us access to a raft of existing results and tools that have been developed in the Petri net community.

\section{Petri nets}

In this section, we introduce Petri nets with weighted arcs [12], and give their operational semantics in terms of step sequences ${ }^{2}$.

A (weighted) net is a triple $N=(P, T, W)$ such that $P$ and $T$ are disjoint finite sets, and $W:(T \times P) \cup(P \times T) \rightarrow \mathbb{N}$. The elements of $P$ and $T$ are respectively the places and transitions, and $W$ is the weight function of $N$. In diagrams, places are drawn as circles, and transitions as rectangles. If $W(x, y) \geq 1$ for some $(x, y) \in(T \times P) \cup(P \times T)$, then $(x, y)$ is an arc leading from $x$ to $y$. As usual, arcs are annotated with 
their weight if this is 2 or more. We assume that, for every $t \in T$, there is a place $p$ such that $W(p, t) \geq 1$.

The pre-and post-multiset of a transition $t \in T$ are multisets of places, $\operatorname{PRE}_{N}(t)$ and $\operatorname{POST}_{N}(t)$, respectively given by $\operatorname{PRE}_{N}(t)(p)=W(p, t)$ and $\operatorname{PosT}_{N}(t)(p)=W(t, p)$, for all $p \in P$. Both notations extend to finite multisets of transitions $U: \operatorname{PRE}_{N}(U)=\sum_{t \in U} U(t) \cdot \operatorname{PRE}_{N}(t)$ and $\operatorname{POST}_{N}(U)=\sum_{t \in U} U(t) \cdot \operatorname{POST}_{N}(t)$.

A marking of a net $N$ is a multiset of places. Following the standard terminology, given a marking $M$ of $N$ and a place $p \in P$, we say that $p$ is marked if $M(p) \geq 1$ and that $M(p)$ is the number of tokens in $p$. In diagrams, $M$ will be represented by drawing in each place $p$ exactly $M(p)$ tokens (black dots).

Transitions represent actions which may occur at a given marking and then lead to a new marking. Here we define this dynamics in terms of multisets of (simultaneously occurring) transitions.

A step is a non-empty finite multiset of transitions, $U: T \rightarrow \mathbb{N}$. It is enabled at a marking $M$ if $M \geq \operatorname{PRE}_{N}(U)$. Thus, in order for $U$ to be enabled at $M$, for each place $p$, the number of tokens in $p$ under $M$ should at least be equal to the total number of tokens that are needed as an input to $U$, respecting the weights of the input arcs.

If $U$ is enabled at $M$, then it can be executed leading to the marking $M^{\prime}=M-\operatorname{PRE}_{N}(U)+\operatorname{POST}_{N}(U)$. This means that the execution of $U$ 'consumes' from each place $p$ exactly $W(p, t)$ tokens for each occurrence of a transition $t \in U$ that has $p$ as an input place, and 'produces' in each place $p$ exactly $W(t, p)$ tokens for each occurrence of a transition $t \in U$ with $p$ as an output place. If the execution of $U$ leads from $M$ to $M^{\prime}$ we write $M[U\rangle M^{\prime}$.

An execution from a marking $M$ to a marking $M^{\prime}$ is a sequence $\mu=$ $M U_{1} M_{1} \ldots M_{n-1} U_{n} M^{\prime}$ such that $M\left[U_{1}\right\rangle M_{1} \cdots M_{n-1}\left[U_{n}\right\rangle M^{\prime}$. We also say that $M^{\prime}$ is reachable from $M$.

\section{Observing Petri net behaviour}

In this section, we introduce a specific device aimed at modelling various observation capabilities based on the executed behaviours of a Petri net. Our framework is deliberately general to allow one to deal with a wider range of observation scenarios. We also extend the previous scheme, by allowing even greater discriminating power on the observer's side.

We start by making a small (but important from the point of view of applications) adjustment of the standard notion of a marked net, by assuming that the system specification we are given at the outset is a 
pair $\Sigma=\left(N, \mathcal{M}_{0}\right)$, where $N$ is a net as defined in the previous section and $\mathcal{M}_{0}$ is a non-empty finite set of initial markings. This allows us to easily model situations where only partial information of the initial state of the system is available to an observer.

We will denote by $\left[\mathcal{M}_{0}\right\rangle$ the set of all markings reachable from any of the markings in $\mathcal{M}_{0}$, and by $R G(\Sigma)$ the reachability graph of $\Sigma$ defined as the labelled directed graph whose nodes are the markings in $\left[\mathcal{M}_{0}\right\rangle$, and the labelled arcs represent all steps executed at these markings according to the rules from the previous section We will denote by $R G_{\text {steps }}(\Sigma)$ the set of all the steps labelling the arcs of $R G(\Sigma)$.

\subsection{Visibility of reachable markings and executed steps}

In our approach, we assume that there is a mapping obs which for each reachable marking in $\left[\mathcal{M}_{0}\right\rangle$ returns some label $o b s(M)$ which is meant to capture the observable or visible aspects of system's global states. We further assume that the mapping is defined for steps of executed transitions; more precisely, for each reachable marking $M$ and a step of transitions $U$ enabled by $M, o b s(M, U)$ issome label which is meant to capture the observable or visible aspects of executing step $U$ at the global state $M$.

We do not place any restrictions on the nature of the obs mapping at this point; indeed, it is left under-specified deliberately to accommodate a wide range of observation scenarios. We only assume that markings and steps are visible through different sets of labels (i.e., obs $(M) \neq$ $\operatorname{obs}\left(M^{\prime}, U\right)$, for all $M, M^{\prime} \in\left[\mathcal{M}_{0}\right\rangle$ and $U$ enabled at $\left.M^{\prime}\right)$.

We employ a special label $\tau$ which is returned as the value of $o b s(M, U)$ in cases when $U$ is a step invisible to the observer in the system state $M$.

Notice that, unlike in [1], we do not define the mapping obs for steps, i.e., $o b s(U)$ is not assumed to be given (clearly, if $o b s(M, U)$ returns the same label $\ell$ for all markings $M$ enabling $U$, then we can define $o b s(U)=\ell$ and we have exactly the setup from [1]). The motivation for this is that we envisage application when the observability of executed transitions would depend on the current state of the system (for example, after breaking one of the cryptographic keys used by a system under attack, the adversary would typically be able to deduce more from the observed message exchange).

Suitable choices of obs mapping can be used to encode the various levels of visibility of system behaviour that we attribute to the environment or adversary. Thus transitions visible only to a secret user might 
be mapped to a $\tau$ label. Such events would be completely invisible to the environment, i.e., the environment would not be aware that any transition had occurred (and, if all the transitions in an executed step are invisible, then the whole step is mapped to $\tau$ ). Transitions corresponding to the transmission of encrypted values could be mapped to a single label. Transitions deemed visible to the adversary may be left unchanged by the obs mapping.

Note that, in particular, obs allows us to 'detect' properties like deadlock-freeness or acceptance sets. The theory is rich enough to incorporate and reason about them. It is another matter, of course, how deadlocks would be detected or observed in the real life system, but these issues are beyond the scope of the current paper.

Having defined the observable aspects of individual markings and steps of transitions, we can define the effect of the observation mapping on the executions of the marked net $\Sigma$.

Let $\mu=M_{0} U_{1} M_{1} \ldots M_{n-1} U_{n} M_{n}$ be an execution from a marking $M_{0} \in \mathcal{M}_{0}$. We first introduce two auxiliary notations:

- $o b s^{\prime}(\mu)=\ell_{0} \ell_{1}^{\prime} \ell_{1} \ldots \ell_{n}^{\prime} \ell_{n}$ is the sequence obtained from $\mu$ by replacing each $M_{i}$ by $\ell_{i}=o b s\left(M_{i}\right)$ and each $U_{i}$ by $\ell_{i}^{\prime}=o b s\left(M_{i-1}, U_{i}\right)$.

- $o b s^{\prime \prime}(\mu)$ is obtained from $o b s^{\prime}(\mu)$ by replacing each maximal subsequence $\ell_{i} \ell_{i+1}^{\prime} \ell_{i+1} \ldots \ell_{j}^{\prime} \ell_{j}$ such that $i \leq j$ and $\ell_{i}=\cdots=\ell_{j}$ and $\ell_{i+1}^{\prime}=\cdots=\ell_{j}^{\prime}=\tau$, by $\ell_{i}^{S}$, where $S=\left\{M_{i}, M_{i+1}, \ldots, M_{j}\right\}$.

$o b s^{\prime \prime}$ collapses sequences of the same (observable) states interspersed with $\tau$ 's into a single state, since this is what will be observable to the user.

Suppose now that $o b s^{\prime \prime}(\mu)=\hat{\ell}_{1}^{S_{1}} \hat{\ell}_{1}^{\prime} \hat{\ell}_{2}^{S_{2}} \ldots \hat{\ell}_{m}^{S_{m}}$. Then the observation of $\mu$ is given by obs $(\mu)=\hat{\ell}_{1} \hat{\ell}_{1}^{\prime} \hat{\ell}_{2} \ldots \hat{\ell}_{m}$. Moreover, for each $i \leq m, o b s^{i}(\mu)=$ $S_{i}$ and obs $^{\text {init }}(\mu)=S_{1}$, obs ${ }^{\text {fin }}(\mu)=S_{m}$, obs ${ }^{\text {all }}(\mu)=S_{1} \cup \ldots \cup S_{m}$.

For example, if $\mu=M_{0} U_{1} M_{1} U_{2} M_{2} U_{3} M_{3}$ is such that $\operatorname{obs}^{\prime}(\mu)=$ $z \tau z b w \tau y$ then $\operatorname{obs}(\mu)=z b w \tau y$, obs ${ }^{\text {init }}(\mu)=\left\{M_{0}, M_{1}\right\}$, obs $^{\text {fin }}(\mu)=$ $\left\{M_{3}\right\}$ and obs ${ }^{\text {all }}(\mu)=\left\{M_{0}, M_{1}, M_{2}, M_{3}\right\}$.

Note that one could have deleted from $o b s(\mu)$ all the remaining $\tau$ 's without changing any of the subsequent results, but this would have led to a more complicated definitions and constructions in proofs.

Examples. The two basic forms of defining the obs mapping are transition labelling and marking projection. In the first case, we assume that each transition $t$ has its own (not necessarily unique) label $\ell(t)$ and 
then the visibility of a step $U=\left\{t_{1}, \ldots, t_{k}\right\}$ is defined as the multiset

$$
\ell(U)= \begin{cases}\tau & \text { if } \ell\left(t_{1}\right)=\cdots=\ell\left(t_{k}\right)=\tau \\ \left\{\ell\left(t_{i}\right) \mid \ell\left(t_{i}\right) \neq \tau\right\} & \text { otherwise }\end{cases}
$$

In the case of marking projection, we assume that $V i s \subseteq P$ is a set of places on which we can always see the tokens, and all places in $P \backslash V i s$ are hidden from us (in the extreme case, Vis $=\varnothing$ which effectively means that no information about the tokens is available). Then, for every marking $M$, we define $\left.M\right|_{V i s}$ as a multiset over Vis such that $\left.M\right|_{V i s}(p)=M(p)$ for every place $p \in V i s$.

Dynamic observation functions can be encoded using these projections. We simply include information that the observer has as part of the net. For example, in Figure 1, the net on the left represents the transmitting of messages encrypted using a key $k$. The net on the right represents the observers state of knowledge of the encryption key. Before the inverse key $\left(k^{-1}\right)$ is known is represented by state $S_{2}$ and after the inverse key is known by state $S_{3}$. The key may be refreshed (modelled by the ref transition); this moves the observer back to the initial state. We include the possibilty that the ref transition may also occur before the key is compromised.

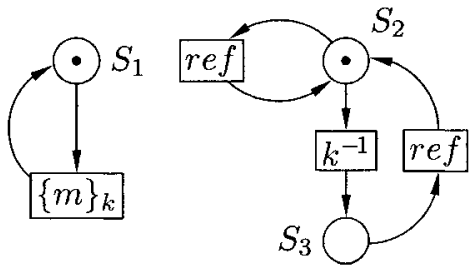

Figure 1. An encoding of a dynamic observation mapping.

The (relevant part of the) obs mapping can then be defined as $o b s(M)=M, o b s\left(\left\{S_{1}, S_{2}\right\},\{m\}_{k}\right)=\{m\}_{k}, o b s\left(\left\{S_{1}, S_{3}\right\},\{m\}_{k}\right)=m$ and so when the observer has the inverse key he can read any messages which pass on this channel.

Of course, once the observer has the inverse key he can go back to any previously read messages which he has saved, and decode them offine. It is possible that the observation function could also take this posthoc analytic ability into consideration, but we do not pursue that idea further in this paper.

The same construction also seems suitable to model the notion of a downgrader: Once the appropriate downgrade action takes place an 
uncleared user may observe the messages or read the files which have been downgraded.

Figure 2 is an example of using the $\tau$ transitions to model information flow. Consider two system users, high and low. high is able to execute either of two large processes, initiating them with the action $e^{x} e_{1}$ or $e^{2 x e_{2}}$ as appropriate. low is using the same system, but is a lower-priority user. His work request will be disallowed if high is executing one or other of his processes.

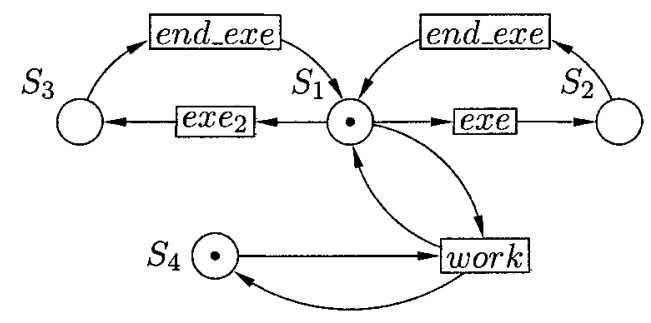

Figure 2. An encoding of an information flow.

We can consider the $e x e_{1}$ and $e x e_{2}$ transitions as silent or $\tau$ transitions with respect to the low user. If the high user is in state $S_{2}$ or $S_{3}$ then when low attempts to work he will be unable to perform the transition. This will allow him to deduce that either $S_{2}$ or $S_{3}$ is occupied, but not which one.

The (relevant part of) the obs mapping would then be defined as

$$
\begin{aligned}
\text { obs }(M) & =\left.M\right|_{S_{4}} \\
\operatorname{obs}\left(\left\{S_{1}, S_{4}\right\},\{\text { work }\}\right) & =\text { work } \\
\operatorname{obs}\left(\left\{S_{2}, S_{4}\right\},\{\text { exe }\}\right) & =\tau \\
\operatorname{obs}\left(\left\{S_{3}, S_{4}\right\},\{\text { exe }\}\right) & =\tau \\
\operatorname{obs}\left(\left\{S_{2}, S_{4}\right\},\{\text { end_exe }\}\right) & =\tau \\
\text { obs }\left(\left\{S_{3}, S_{4}\right\},\{\text { end_exe }\}\right) & =\tau
\end{aligned}
$$

Although low cannot see the state of high directly, some information can be deduced by the willingness of the system to respond to his own work requests. Either high is in state $S_{1}$ and the work request will be granted, or high is in one of states $S_{2}$ and $S_{3}$, and the work request will not be granted.

In fact, this approach appears somewhat linked to the CSP refusals semantic model [9]. In that, the semantics of the system is taken to 
be the observed traces of events, together with the sets of events that may be refused to an observer. Here, the semantics of the systems (as observed by low) is taken as a trace of states and transitions (events) but some refusal information is captured by our treatment of silent actions.

Exploring and formalising such links to other semantic models is not pursued here, but will be considered in a forthcoming paper.

\section{$3.2 \quad$ Opacity}

In the present framework, we are interested in whether an observer can establish a property $\mathcal{P}$ at some specific state(s) of the execution of the system solely on the basis of its visible version. We consider here any state property, i.e., one which can be evaluated at any reachable marking in $\left[\mathcal{M}_{0}\right\rangle$. Clearly, any such property can simply be represented as the set of those reachable markings where it holds, and so we will take $\mathcal{P}$ to be any subset of $\left[\mathcal{M}_{0}\right\rangle$.

Now, given an observed execution of the system, we will be interested in finding out whether the fact that an underlying marking belongs to $\mathcal{P}$ can be deduced by the observer. Note, however, that we are not interested in establishing whether the underlying marking does not belong to $\mathcal{P}$. To do this, we would rather consider the property $\overline{\mathcal{P}}=\left[\mathcal{M}_{0}\right\rangle \backslash \mathcal{P}$.

What it means to deduce a property can mean different things depending on what is relevant or important from the point of view of real application. Below, we formalise four possible ways of defining variants of opacity. The first two properties can be used to capture the fact that we are only interested in the holding of our property in the observed initial or final state, respectively.

- $\mathcal{P}$ is initial-opaque if for every execution $\mu$ from any marking in $\mathcal{M}_{0}$, if $o b s^{\text {init }}(\mu) \cap \mathcal{P} \neq \varnothing$, then there exists an execution $\mu^{\prime}$ from a marking in $\mathcal{M}_{0}$ such that obs $(\mu)=o b s\left(\mu^{\prime}\right)$ and obs ${ }^{i n i t}\left(\mu^{\prime}\right) \cap \mathcal{P}=\varnothing$.

- $\mathcal{P}$ is final-opaque if for every execution $\mu$ from any marking in $\mathcal{M}_{0}$, if $o b s^{f i n}(\mu) \cap \mathcal{P} \neq \varnothing$, then there exists an execution $\mu^{\prime}$ from a marking in $\mathcal{M}_{0}$ such that obs $(\mu)=o b s\left(\mu^{\prime}\right)$ and $o b s^{f i n}\left(\mu^{\prime}\right) \cap \mathcal{P}=\varnothing$.

The next property reflects a view that we are interested in the holding of our property at all the specific observed states of the execution.

- $\mathcal{P}$ is always-opaque if for every execution $\mu$ from any marking in $\mathcal{M}_{0}$, if $o b s^{i}(\mu) \cap \mathcal{P} \neq \varnothing$ for some $i$, then there exists an execution $\mu^{\prime}$ from a marking in $\mathcal{M}_{0}$ such that obs $(\mu)=o b s\left(\mu^{\prime}\right)$ and $o b s^{i}\left(\mu^{\prime}\right) \cap$ $\mathcal{P}=\varnothing$.

The last property capture the situation that the holding of our property can never be established for sure. 
- $\mathcal{P}$ is total-opaque if for every execution $\mu$ from any marking in $\mathcal{M}_{0}$ such that $\operatorname{obs}^{\text {all }}(\mu) \cap \mathcal{P} \neq \varnothing$ there exists an execution $\mu^{\prime}$ from a marking in $\mathcal{M}_{0}$ such that $o b s(\mu)=o b s\left(\mu^{\prime}\right)$ and $o b s^{\text {all }}\left(\mu^{\prime}\right) \cap \mathcal{P}=\varnothing$.

initial-opacity is illustrated by the dining cryptographers example. It would appear that initial-opacity is suited to modelling situations in which initialisation information such as crypto keys, etc., needs to be kept secret. More generally, situations in which confidential information can be modelled in terms of initially resolved non-determinism can be captured in this way. always-opacity would seem more appropriate to capture situation in which secret information is input at run time, for example due to high level interactions.

The distinction between total-opacity and always-opacity can be illustrated in the two diagrams below.

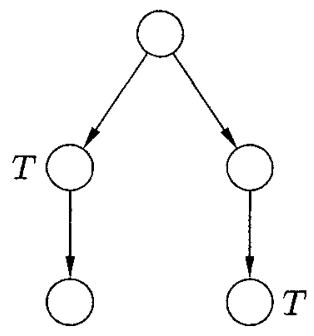

(a)

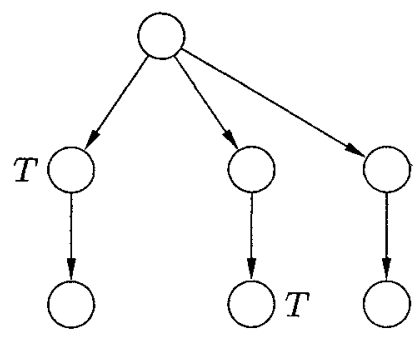

(b)

Figure 3. The difference between total-opacity and final-opacity.

Assume that each of the paths through the nets in figure 3 gives rise to the same observation. In figure 3(a), a property which holds at each of the states marked $T$ will be always-opaque, because at each of the states where it holds there is a corresponding execution for which the property does not hold in the corresponding state. But it is not total-opaque, because by the end of whichever execution we observe, we can say that the property has held at some point. For total-opacity we would require the extra execution of figure 3(b). Now, at the end of the execution we do not know if the property was ever true.

Informally, the notion of non-inference, [11], captures the idea that an observer should never be able to eliminate the possibility that the High user did nothing. More formally:

$$
\begin{aligned}
& \forall t \in \operatorname{traces}(S) \quad \exists t^{\prime} \in \operatorname{traces}(S) \bullet \\
& t \downarrow \text { Low }=t^{\prime} \downarrow \text { Low } \wedge t^{\prime} \downarrow H i g h=\langle\rangle
\end{aligned}
$$


We will be exploring the application of these formal properties in a future paper.

PROPOSITION 1 If $\mathcal{P}$ is total-opaque then it is initial-opaque, final-opaque and always-opaque. Moreover, if $\mathcal{P}$ is always-opaque then it is initialopaque and final-opaque. No other implication of this kind in general holds.

TheOREM 2 For $x \in\{$ initial, fnal, always, total $\}$, it is the case that $\mathcal{P}=\varnothing$ is $x$-opaque, $\mathcal{P}=\left[\mathcal{M}_{0}\right\rangle$ is not $x$-opaque, and if $\mathcal{P} \subseteq \mathcal{P}^{\prime}$ and $\mathcal{P}^{\prime}$ is $x$-opaque then $\mathcal{P}$ is $x$-opaque.

What now follows are crucial results stating that three of the four notions of opacity are decidable provided that the system has finitely many states. In all the results that follow, it is assumed that $\left[\mathcal{M}_{0}\right\rangle$ is finite. ${ }^{3}$

THEOREM 3 It is decidable whether $\mathcal{P}$ is initial-opaque.

THEOREM 4 It is decidable whether $\mathcal{P}$ is final-opaque.

THEOREM 5 It is decidable whether $\mathcal{P}$ is total-opaque.

\section{Dining cryptographers}

To illustrate our approach, we use the example of the dining cryptographers, first presented in [4]. A simplified version (involving only two cryptographers) was presented in [1].

This example involves three diners and admits some further anonymity properties, e.g., a paying cryptographer can remain anonymous w.r.t. his or her companions.

The three cryptographers, Anne, Bob and Charlie, enjoy a meal in a restaurant. When they call for the bill, the waiter tells them that it has already been paid. Each cryptographer wishes to know whether the bill was paid by the NSA, or if it was one of them. However, if one of them paid, they do not want an eavesdropper, Yves, on the neighbouring table to know which of them paid. The protocol they choose to solve this problem is as follows:

They each toss a coin, and reveal the result only to the cryptographer sitting one their left. Yves, of course, cannot see any of the coins. If Anne paid, she lies about the parity of the two coins (she calls 'agree' if she sees a head and a tail, and 'disagree' otherwise). If Anne did not pay, she tells the truth about the parity of the coins. Similarly for Bob and Charlie. Now each cryptographer knows if the NSA paid for the meal, 
or if the bill was settled by one of them. If the number of "disagree" calls is even, then the NSA is paying. If the number is odd, then one of the cryptographers is paying. In this case, the other two cryptographers do not know which of their dining companions has paid for the meal. Yves cannot distinguish the paying cryptographer from the others.

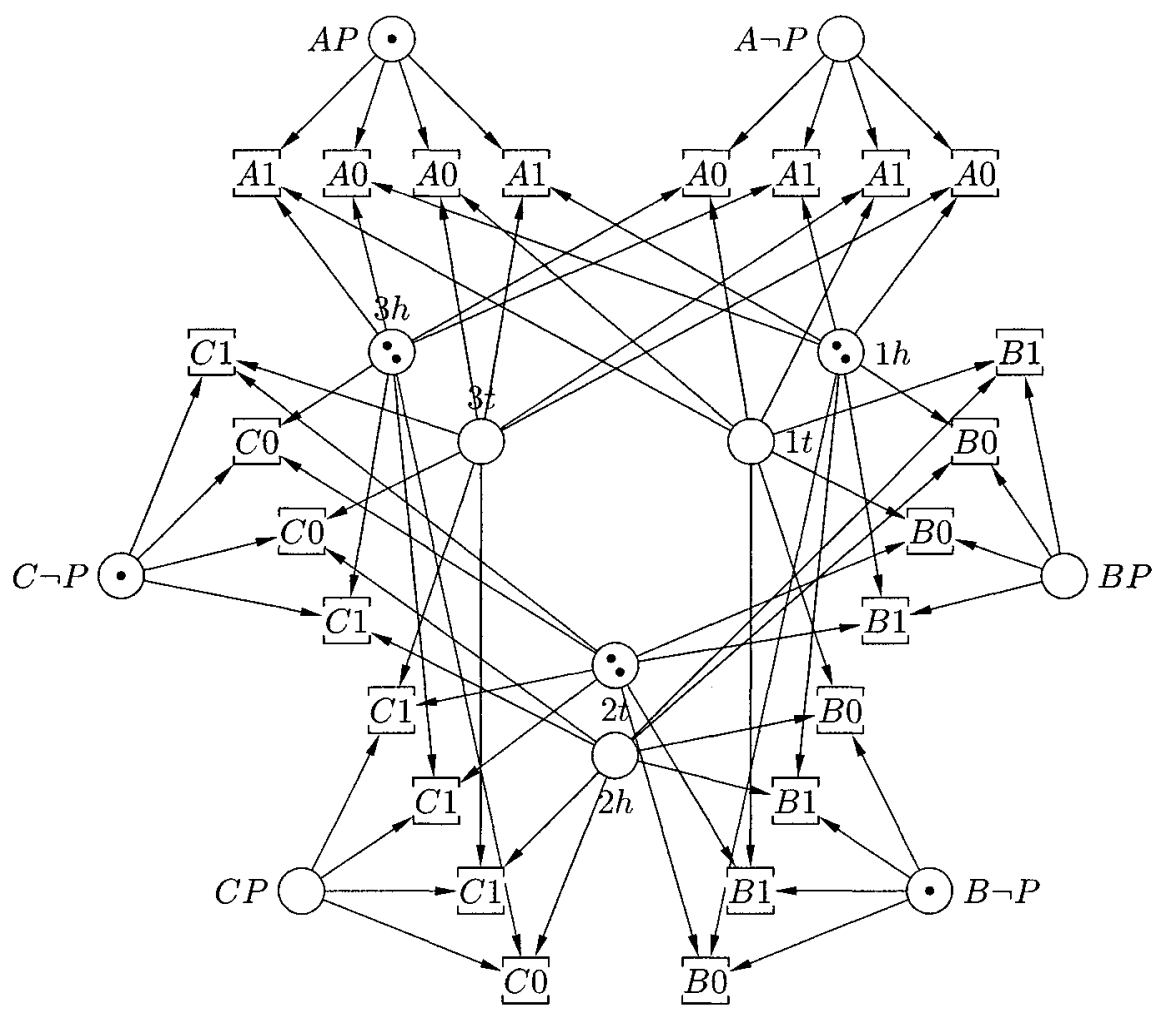

Figure 4. Net for the 3-way dining cryptographers example with one of the $n$ initial markings.

Figure 4 presents a possible encoding of the protocol. The two places at the top of the diagram represent Anne's initial state (having paid is represented by placing a single token in place $A P$, and having not paid is represented by placing a single token in place $A \neg P$ ). The two places at the right represent Bob's initial state $(B P$ and $B \neg P)$ and the two places on the left Charlie's initial state $(C P$ and $C \neg P)$. The possible initial markings for these places are $\{A P, B \neg P, C \neg P\},\{A \neg P, B P, C \neg P\}$, $\{A \neg P, B \neg P, C P\},\{A \neg P, B \neg P, C \neg P\}$. The three sets of two places in 
the centre of the diagram represent the three coins (heads is represented by placing tokens in place $c_{i} h$, and tails is represented by placing tokens in place $c_{i} t$, for $\left.i=1,2,3\right)$. For each pair, the marked place must contain two tokens. This is because two cryptographers must see each coin. The possible initial markings for the coins are therefore

$\{\{1 h, 1 h\},\{1 t, 1 t\}\} \times\{\{2 h, 2 h\},\{2 t, 2 t\}\} \times\{\{3 h, 3 h\},\{3 t, 3 t\}\}$

The set of possible initial markings, $\mathcal{M}_{0}$, is the cross product of the cryptographer markings and the coin markings.

The eight transitions at the top represent the eight possible scenarios for Anne, given by two possibilities for each coin multiplied by the two possibilities for her own initial state. Each transition is labelled with ' $A 0$ ' (if Anne says the coins 'disagree') or ' $A 1$ ' (if Anne says the coins 'agree'). Similarly for Bob on the right and Charlie on the left. This gives the transition labelling $\ell$ which will be used for defining the visibility of steps.

Yves' observation function is simple. He can see none of the places (he does not know the initial state of the cryptographers, nor the state of the coins), but he can see all of the labels of the executed transitions (he can hear all that they say). In other words, for every reachable marking $M$ and executed step $U$, we have the following (see section 3.1):

$o b s_{Y}(M)=\left.M\right|_{\varnothing}=\varnothing, o b s_{Y}(U)=\ell(U)$.

Note that Yves also knows the structure of the original net, i.e., the protocol.

We wish to demonstrate that after observing the execution of transitions, although Yves may be able to determine whether the meal was paid for by one of the cryptographers, he can never know which one. The three properties we wish to be initial-opaque are therefore $\mathcal{P}_{A}=\left\{M \in \mathcal{M}_{0} \mid M(A P)=1\right\}$ and $\mathcal{P}_{B}=\left\{M \in \mathcal{M}_{0} \mid M(B P)=1\right\}$. $\mathcal{P}_{C}=\left\{M \in \mathcal{M}_{0} \mid M(C P)=1\right\}$.

Yves cannot determine the satisfaction of any of the above predicates. Note, however, that Yves can in either case determine the satisfaction of the property $\mathcal{P}=\left\{M \in \mathcal{M}_{0} \mid M(A P)+M(B P)+M(C P)=1\right\}$, i.e., he knows when one of the cryptographers paid the bill. In terms of our framework, both $\mathcal{P}_{1}$ and $\mathcal{P}_{2}$ are initial-opaque, but $\mathcal{P}$ is not.

These properties are similar to the ones which hold for the limited version of the case study, presented in [1]. The difference is that in the situation where one of the cryptographers paid for the meal, the other two do not know who it was. The paying cryptographer remains anonymous with respect to their dining companions. To see this, we model the point of view of one of the cryptographers, by change the obs function to model the increased level of knowledge. For example, the observation function of Anne is such that, for every reachable marking 
$M$ and executed step $U$,

$$
\begin{aligned}
o b s_{\text {Anne }}(M) & =\left.M\right|_{\{A P, A \neg P, 1 h, 1 t, 3 h, 3 t\}} \\
o b s_{\text {Anne }}(U) & =\ell(U) .
\end{aligned}
$$

Anne knows her own initial state, and can see the state of her coin and the coin on her left. Given this observation function, she learns exactly what she wants to know - did the NSA pay, or was it one of her friends?

\section{Conclusions and future work}

We have presented an extension of our earlier Petri net framework in order be able to capture a richer class of information flow requirements. In this richer model we can encompass conditional information flow policies (e.g., downgraders) as well as scenarios that include key compromise and key refreshment.

In this paper we model the full dining cryptographers example which allows us to illustrate how various flavours of anonymity can be captured, corresponding to the various observer viewpoints.

A further advance presented here is a full treatment of invisible events.

The decidability results of the earlier paper have been extended to the richer model presented here and for the new total opacity property.

In future work we intend to explore the relationship of the approach presented here to process algebraic formulations of generalised noninterference [14] and anonymity [15].

A major challenge in such work is the choice of appropriate abstractions to encode the adversary's observational capabilities. This is particularly delicate where cryptographic mechanisms are involved. Adversary deductions and algebraic manipulations complicate the modelling. We intend to investigate using the dynamic obs mappings presented here to address such issues.

A further line of research is to explore analogues in this framework of the notion of non-deducibility on strategies, due to Johnson and Wittbold [17]. This seeks to capture the possibility of a secret user and an uncleared user colluding and using adaptive strategies to cause information flows in violation of the policy. This is likely to require more precise modelling of various flavours of non-determinism within the Petri net framework.

We will also investigate the problem of preservation of opacity properties under refinement and composition of Petri nets.

\section{Acknowledgement}

This research was supported by the EPSRC GOLD project and DSTL. 


\section{Notes}

1. A fuller version of this paper, which includes the omitted proofs, can be found at [2].

2. We have already demonstrated in [1] that step and interleaving semantics lead to different notions of opacity (even without the $\tau$ labels we introduce later).

3. Note that the finiteness of $\left[\mathcal{M}_{0}\right]$ is decidable, and can be checked using the standard coverability tree construction [12].

\section{References}

[1] J.W.Bryans, M.Koutny and P.Y.A.Ryan: Modelling Opacity using Petri Nets. Proceedings of WISP 2004 (2004)

[2] J.W.Bryans, M.Koutny and P.Y.A.Ryan: Modelling Dynamic Opacity using Petri Nets with Silent Actions University of Newcastle Technical Report Series 2004

[3] N.Busi and R.Gorrieri: Structural Non-interference with Petri Nets. Proceedings of WITS 2004 (2004)

[4] D. Chaum: The dining cryptographers problem: unconditional sender and receipient untraceability. Journal of Cryptology, 1, 1988

[5] E.Cohen: Information Transmission in Computational Systems. Proceedings of 6th ACM Symposium on Operating System Principles (1997)

[6] R.J.Feiertag: A technique for Proving Specifications are Multi-level Secure Technical. Report CSL109, CSL, SRI International (1980)

[7] J.Goguen and J.Meseguer: Security Policies and Security Models. Proceedings of IEEE Symposium on Security and Privacy (1982)

[8] J.Goguen and J.Meseguer: Inference Control and Unwinding. Proceedings of IEEE Symposium on Research in Security and Privacy (1984)

[9] C. A. R. Hoare: Communicating Sequential Processes (1985)

[10] L. Mazare: Using Unification for Opacity Properties. Proceedings of WITS 2004 (2004)

[11] C. O'Halloran: A Calculus of Information Flow. Proceedings of ESORICS (1990)

[12] W.Reisig and G.Rozenberg (Eds.): Lectures on Petri Nets. LNCS 1491 \& 1492 (1998)

[13] J.Rushby: Noninterference, Transitivity and Channel-Control Security Policies. SRI Techical Report (1992)

[14] P.Y.A.Ryan: Mathematical Models of Computer Security. Proceedings of Foundations of Security Analysis and Design, LNCS 2171 (2000)

[15] S.A.Schneider and A.Sidiropoulos: CSP and Anonymity. Proceedings of ESORICS (2000)

[16] D.Sutherland: A Model of Information, Proceedings of 9th National Computer Security Conference (1986)

[17] J.T.Wittbold and D.M.Johnson: Information Flow in Nondeterministic Systems. Proceedings of the Symposium on Research on Security and Privacy (1990) 